\title{
On the optimality of gluing over scales
}

\author{
Alexander Jaffe* James R. Lee* Mohammad Moharrami*
}

\begin{abstract}
We show that for every $\alpha>0$, there exist $n$-point metric spaces $(X, d)$ where every "scale" admits a Euclidean embedding with distortion at most $\alpha$, but the whole space requires distortion at least $\Omega(\sqrt{\alpha \log n})$. This shows that the scale-gluing lemma [Lee, SODA 2005] is tight, and disproves a conjecture stated there. This matching upper bound was known to be tight at both endpoints, i.e. when $\alpha=\Theta(1)$ and $\alpha=\Theta(\log n)$, but nowhere in between.

More specifically, we exhibit $n$-point spaces with doubling constant $\lambda$ requiring Euclidean distortion $\Omega(\sqrt{\log \lambda \log n})$, which also shows that the technique of "measured descent" [Krauthgamer, et. al., Geometric and Functional Analysis] is optimal. We extend this to $L_{p}$ spaces with $p>1$, where one requires distortion at least $\Omega\left((\log n)^{1 / q}(\log \lambda)^{1-1 / q}\right)$ when $q=\max \{p, 2\}$, a result which is tight for every $p>1$.
\end{abstract}

\section{Introduction}

Suppose one is given a collection of mappings from some finite metric space $(X, d)$ into a Euclidean space, each of which reflects the geometry at some "scale" of $X$. Is there a non-trivial way of gluing these mappings together to form a global mapping which reflects the entire geometry of $X$ ? The answers to such questions have played a fundamental role in the best-known approximation algorithms for Sparsest Cut [7, 10, 4, 1] and Graph Bandwidth [17, 17, 11, and have found applications in approximate multi-commodity max-flow/min-cut theorems in graphs [17, 17. In the present paper, we show that the approaches of [7] and [10] are optimal, disproving a conjecture stated in [10].

Let $(X, d)$ be an $n$-point metric space, and suppose that for every $k \in \mathbb{Z}$, we are given a nonexpansive mapping $\phi_{k}: X \rightarrow L_{2}$ which satisfies the following. For every $x, y \in X$ with $d(x, y) \geq 2^{k}$, we have

$$
\left\|\phi_{k}(x)-\phi_{k}(y)\right\| \geq \frac{2^{k}}{\alpha}
$$

The Gluing Lemma of [10] (generalizing the approach of [7]) shows that the existence of such a collection $\left\{\phi_{k}\right\}$ yields a Euclidean embedding of $(X, d)$ with distortion $O(\sqrt{\alpha \log n})$. (See Section 1.1 for the relevant definitions on embeddings and distortion.) This is known to be tight when $\alpha=\Theta(1)$ [16] and also when $\alpha=\Theta(\log n)$ [13, 2], but nowhere in between. In fact, in [10], the second named author conjectured that one could achieve $O(\alpha+\sqrt{\log n})$ (this is indeed stronger, since one can always construct $\left\{\phi_{k}\right\}$ with $\left.\alpha=O(\log n)\right)$.

In the present paper, we give a family of examples which shows that the $\sqrt{\alpha \log n}$ bound is tight for any dependence $\alpha(n)=O(\log n)$. In fact, we show more. Let $\lambda(X)$ denote the doubling

\footnotetext{
*Research partially supported by NSF CCF-0644037 and a Sloan Research Fellowship.
} 
constant of $X$, i.e. the smallest number $\lambda$ so that every open ball in $X$ can be covered by $\lambda$ balls of half the radius. In [7, using the method of "measure descent," the authors show that $(X, d)$ admits a Euclidean embedding with distortion $O(\sqrt{\log \lambda(X) \log n})$. (This is a special case of the Gluing Lemma since one can always find $\left\{\phi_{k}\right\}$ with $\alpha=O(\log \lambda(X))$ [5]). Again, this bound was known to be tight for $\lambda(X)=\Theta(1)$ [8, 9, 5] and $\lambda(X)=n^{\Theta(1)}$ [13, 2], but nowhere in between. We provide the matching lower bound for any dependence of $\lambda(X)$ on $n$. We also generalize our method to give tight lower bounds on $L_{p}$ distortion for every fixed $p>1$.

Construction and analysis. In some sense, our lower bound examples are an interpolation between the multi-scale method of [16] and [8], and the expander Poincaré inequalities of [13, 2, 14]. We start with a vertex-transitive expander graph $G$ on $m$ nodes. If $D$ is the diameter of $G$, then we create $D+1$ copies $G^{1}, G^{2}, \ldots, G^{D+1}$ of $G$ where $u \in G^{i}$ is connected to $v \in G^{i+1}$ if $(u, v)$ is an edge in $G$, or if $u=v$. We then connect a vertex $s$ to every node in $G^{1}$ and a vertex $t$ to every node in $G^{D+1}$ by edges of length $D$. This yields the graph $\vec{G}$ described in Section 2.2 ,

In Section 3, we show that whenever there is a non-contracting embedding $f$ of $\vec{G}$ into $L_{2}$, the following holds. If $\gamma=\frac{\|f(s)-f(t)\|}{d_{\vec{G}}(s, t)}$, then some edge of $\vec{G}$ gets stretched by at least $\sqrt{\gamma^{2}+\Omega(\log m)^{2}}$, i.e. there is a "stretch increase." This is proved by combining the uniform convexity of $L_{2}$ (i.e. the Pythagorean theorem), with the well-known contraction property of expander graphs mapped into Hilbert space. To convert the "average" nature of this contraction to information about a specific edge, we symmetrize the embedding over all automorphisms of $G$ (which was chosen to be vertex-transitive).

To exploit this stretch increase recursively, we construct a graph $\vec{G}^{\oslash k}$ inductively as follows: $\vec{G}^{\ominus k}$ is formed by replacing every edge of $\vec{G}^{\ominus k-1}$ by a copy of $\vec{G}$ (see Section 2.1 for the formal definitions). Now a simple induction shows that in a non-contracting embedding of $\vec{G}^{\ominus k}$, there must be an edge stretched by at least $\Omega(\sqrt{k} \log m)$. In Section 3.1, a similar argument is made for $L_{p}$ distortion, for $p>1$, but here we have to argue about "quadrilaterals" instead of "triangles" (in order to apply the uniform convexity inequality in $L_{p}$ ), and it requires slightly more effort to find a good quadrilateral.

Finally, we observe that if $\widetilde{G}$ is the graph formed by adding two tails of length $3 D$ hanging off $s$ and $t$ in $\vec{G}$, then (following the analysis of [8, 9]), one has $\log \lambda\left(\widetilde{G}^{\ominus k}\right) \lesssim \log m$. The same lower bound analysis also works for $\widetilde{G}^{\ominus k}$, so since $n=\left|V\left(\widetilde{G}^{\ominus k}\right)\right|=2^{\Theta(k \log m)}$, the lower bound is

$$
\sqrt{k} \log m \approx \sqrt{\log m \log n} \gtrsim \sqrt{\log \lambda\left(\widetilde{G}^{\oslash k}\right) \log n}
$$

completing the proof.

\section{$1.1 \quad$ Preliminaries}

For a graph $G$, we will use $V(G), E(G)$ to denote the sets of vertices and edges of $G$, respectively. Sometimes we will equip $G$ with a non-negative length function len $: E(G) \rightarrow \mathbb{R}_{+}$, and we let $d_{\text {len }}$ denote the shortest-path (semi-)metric on $G$. We refer to the pair ( $G$, len) as a metric graph, and often len will be implicit, in which case we use $d_{G}$ to denote the path metric. We use Aut $(G)$ to denote the group of automorphisms of $G$.

Given two expressions $E$ and $E^{\prime}$ (possibly depending on a number of parameters), we write $E=O\left(E^{\prime}\right)$ to mean that $E \leq C E^{\prime}$ for some constant $C>0$ which is independent of the parameters. Similarly, $E=\Omega\left(E^{\prime}\right)$ implies that $E \geq C E^{\prime}$ for some $C>0$. We also write $E \lesssim E^{\prime}$ as a synonym for $E=O\left(E^{\prime}\right)$. Finally, we write $E \approx E^{\prime}$ to denote the conjunction of $E \lesssim E^{\prime}$ and $E \gtrsim E^{\prime}$. 

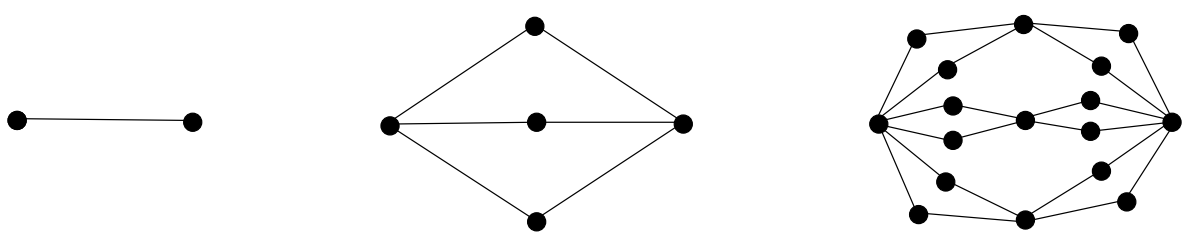

Figure 1: A single edge $H, H \oslash K_{2,3}$, and $H \oslash K_{2,3} \oslash K_{2,2}$.

Embeddings and distortion. If $\left(X, d_{X}\right),\left(Y, d_{Y}\right)$ are metric spaces, and $f: X \rightarrow Y$, then we write

$$
\|f\|_{\text {Lip }}=\sup _{x \neq y \in X} \frac{d_{Y}(f(x), f(y))}{d_{X}(x, y)} .
$$

If $f$ is injective, then the distortion of $f$ is defined by $\operatorname{dist}(f)=\|f\|_{\text {Lip }} \cdot\left\|f^{-1}\right\|_{\text {Lip }}$. A map with distortion $D$ will sometimes be referred to as $D$-bi-lipschitz. If $d_{Y}(f(x), f(y)) \leq d_{X}(x, y)$ for every $x, y \in X$, we say that $f$ is non-expansive. If $d_{Y}(f(x), f(y)) \geq d_{X}(x, y)$ for every $x, y \in X$, we say that $f$ is non-contracting. For a metric space $X$, we use $c_{p}(X)$ to denote the least distortion required to embed $X$ into some $L_{p}$ space.

Finally, for $x \in X, r \in \mathbb{R}_{+}$, we define the open ball $B(x, r)=\{y \in X: d(x, y)<r\}$. Recall that the doubling constant of a metric space $(X, d)$ is the infimum over all values $\lambda$ such that every ball in $X$ can be covered by $\lambda$ balls of half the radius. We use $\lambda(X, d)$ to denote this value.

We now state the main theorem of the paper.

Theorem 1.1. For any positive nondecreasing function $\lambda(n)$, there exists a family of n-vertex metric graphs $\widetilde{G}^{\ominus k}$ such that $\lambda\left(\widetilde{G}^{\ominus k}\right) \lesssim \lambda(n)$, and for every fixed $p>1$,

$$
c_{p}\left(\widetilde{G}^{\oslash k}\right) \gtrsim(\log n)^{1 / q}(\log \lambda(n))^{1-1 / q},
$$

where $q=\max \{p, 2\}$.

\section{Metric construction}

\section{$2.1 \oslash$-products}

An $s$ - $t$ graph $G$ is a graph which has two distinguished vertices $s, t \in V(G)$. For an $s$ - $t$ graph, we use $s(G)$ and $t(G)$ to denote the vertices labeled $s$ and $t$, respectively. We define the length of an $s$ - $t$ graph $G$ as len $(G)=d_{\text {len }}(s, t)$.

Definition 2.1 (Composition of $s$ - $t$ graphs). Given two $s$-t graphs $H$ and $G$, define $H \oslash G$ to be the $s$-t graph obtained by replacing each edge $(u, v) \in E(H)$ by a copy of $G$ (see Figure 1). Formally,

- $V(H \oslash G)=V(H) \cup(E(H) \times(V(G) \backslash\{s(G), t(G)\}))$.

- For every edge $e=(u, v) \in E(H)$, there are $|E(G)|$ edges,

$$
\begin{aligned}
& \left\{\left(\left(e, v_{1}\right),\left(e, v_{2}\right)\right) \mid\left(v_{1}, v_{2}\right) \in E(G) \text { and } v_{1}, v_{2} \notin\{s(G), t(G)\}\right\} \cup \\
& \{(u,(e, w)) \mid(s(G), w) \in E(G)\} \cup\{((e, w), v) \mid(w, t(G)) \in E(G)\}
\end{aligned}
$$


- $s(H \oslash G)=s(H)$ and $t(H \oslash G)=t(H)$.

If $H$ and $G$ are equipped with length functions $\operatorname{len}_{H}$, len ${ }_{G}$, respectively, we define len $=\operatorname{len}_{H \oslash G}$ as follows. Using the preceding notation, for every edge $e=(u, v) \in E(H)$,

$$
\begin{aligned}
\operatorname{len}\left(\left(e, v_{1}\right),\left(e, v_{2}\right)\right) & =\frac{\operatorname{len}_{H}(e)}{d_{\operatorname{len}_{G}}(s(G), t(G))} \operatorname{len}_{G}\left(v_{1}, v_{2}\right) \\
\operatorname{len}(u,(e, w)) & =\frac{\operatorname{len}_{H}(e)}{d_{\operatorname{len}_{G}}(s(G), t(G))} \operatorname{len}_{G}(s(G), w) \\
\operatorname{len}((e, w), v) & =\frac{\operatorname{len}_{H}(e)}{d_{\operatorname{len}_{G}}(s(G), t(G))} \operatorname{len}_{G}(w, t(G)) .
\end{aligned}
$$

This choice implies that $H \oslash G$ contains an isometric copy of $\left(V(H), d_{\operatorname{len}_{H}}\right)$.

Observe that there is some ambiguity in the definition above, as there are two ways to substitute an edge of $H$ with a copy of $G$, thus we assume that there exists some arbitrary orientation of the edges of $H$. However, for our purposes the graph $G$ will be symmetric, and thus the orientations are irrelevant.

Definition 2.2 (Recursive composition). For an $s-t$ graph $G$ and a number $k \in \mathbb{N}$, we define $G^{\oslash k}$ inductively by letting $G^{\oslash 0}$ be a single edge of unit length, and setting $G^{\ominus k}=G^{\oslash k-1} \oslash G$.

The following result is straightforward.

Lemma 2.3 (Associativity of $\oslash$ ). For any three graphs $A, B, C$, we have $(A \oslash B) \oslash C=A \oslash(B \oslash C)$, both graph-theoretically and as metric spaces.

Definition 2.4. For two graphs $G, H$, a subset of vertices $X \subseteq V(H)$ is said to be a copy of $G$ if there exists a bijection $f: V(G) \rightarrow X$ with distortion 1, i.e. $d_{H}(f(u), f(v))=C \cdot d_{G}(u, v)$ for some constant $C>0$.

Now we make the following two simple observations about copies of $H$ and $G$ in $H \oslash G$.

Observation 2.5. The graph $H \oslash G$ contains $|E(H)|$ distinguished copies of the graph $G$, one copy corresponding to each edge in $H$.

Observation 2.6. The subset of vertices $V(H) \subseteq V(H \oslash G)$ form an isometric copy of $H$.

\subsection{A stretched $\vec{G}$}

Let $G=(V, E)$ be an unweighted graph, and put $D=\operatorname{diam}(G)$. We define a metric $s$ - $t$ graph $\vec{G}$ which has $D+1$ layers isomorphic to $G$, with edges between the layers, and a pair of endpoints $s, t$. Formally,

$$
\begin{aligned}
V(\vec{G})= & \{s, t\} \cup\left\{v^{(i)}: v \in V, i \in[D+1]\right\} \\
E(\vec{G})=\left\{\left(s, v^{(1)}\right),\left(v^{(D+1)}, t\right): v \in V\right\} & \\
& \cup\left\{\left(u^{(i)}, v^{(i+1)}\right),\left(u^{(j)}, v^{(j)}\right):(u, v) \in E, i \in[D], j \in[D+1]\right\} \\
& \cup\left\{\left(v^{(i)}, v^{(i+1)}\right): v \in V, i \in[D]\right\} .
\end{aligned}
$$


We put len $\left(s, v^{(1)}\right)=\operatorname{len}\left(v^{(D+1)}, t\right)=D$ for $v \in V, \operatorname{len}\left(u^{(i)}, v^{(i+1)}\right)=\operatorname{len}\left(u^{(j)}, v^{(j)}\right)=1$ for $(u, v) \in E$, $i \in[D], j \in[D+1]$ and $\operatorname{len}\left(v^{(i)}, v^{(i+1)}\right)=1$ for $v \in V, i \in[D]$. We refer to edges of the form $\left(u^{(i)}, v^{(i)}\right)$ as vertical edges. All other edges are called horizontal edges. In particular, there are $D+1$ copies $G^{(1)}, \ldots, G^{(D+1)}$ of $G$ in $\vec{G}$ which are isometric to $G$ itself, and their edges are all vertical.

A doubling version, following Laakso. Let $\vec{G}$ be a stretched graph as in Section 2.2 , with $D=\operatorname{diam}(G)$, and let $s^{\prime}=s(\vec{G}), t^{\prime}=t(\vec{G})$. Consider a new metric $s$ - $t$ graph $\widetilde{G}$, which has two new vertices $s, t$ and two new edges $\left(s, s^{\prime}\right),\left(t^{\prime}, t\right)$ with $\operatorname{len}\left(s, s^{\prime}\right)=\operatorname{len}\left(t^{\prime}, t\right)=3 D$.

Claim 2.7. For any graph $G$ with $|V(G)|=m$, and any $k \in \mathbb{N}$, we have $\log \lambda\left(\widetilde{G}^{\ominus k}\right) \lesssim \log m$.

The proof of the claim is similar to [8, 9], and follows from the following three results.

We define $\operatorname{tri}(G)=\max _{v \in V(G)}\left(d_{\text {len }}(s, v)+d_{\text {len }}(v, t)\right)$. For any graph $G$, we have len $(\widetilde{G})=$ $d(s, t)=9 D$, and it is not hard to verify that $\operatorname{tri}\left(\widetilde{G}^{\oslash k}\right) \leq \operatorname{len}\left(\widetilde{G}^{\ominus k}\right)\left(1+\frac{1}{9 D-1}\right)$. For convenience, let $G_{0}$ be the top-level copy of $\widetilde{G}$ in $\widetilde{G}^{\ominus k}$, and $H$ be the graph $\widetilde{G}^{\ominus k-1}$. Then for any $e \in E\left(G_{0}\right)$, we refer to the copy of $H$ along edge $e$ as $H_{e}$.

Observation 2.8. If $r>\frac{\operatorname{tri}\left(\widetilde{G}^{\ominus k}\right)}{3}$, then the ball $B(x, r)$ in $\widetilde{G}^{\oslash k}$ may be covered by at most $|V(\widetilde{G})|$ balls of radius $r / 2$.

Proof. For any $e \in E\left(G_{0}\right)$, we have $r>\frac{\operatorname{len}(e)}{\operatorname{len}(H)} \operatorname{tri}(H)$, so every point in $H_{e}$ is less than $r / 2$ from an endpoint of $e$. Thus all of $\widetilde{G}^{\ominus k}$ is covered by placing balls of radius $\frac{\operatorname{tri}\left(\widetilde{G}^{\ominus k}\right)}{6}$ around each vertex of $G_{0}$.

Lemma 2.9. If $s \in B(x, r)$, then one can cover the ball $B(x, r)$ in $\widetilde{G}^{\oslash k}$ with at most $|E(\widetilde{G})||V(\widetilde{G})|$ balls of radius $r / 2$.

Proof. First consider the case in which $r>\frac{\operatorname{len}\left(\widetilde{G}^{\ominus k}\right)}{6}$. Then for any edge $e$ in $\widetilde{G}^{\ominus k}$, we have $r>$ $\frac{\operatorname{len}(e)}{\operatorname{len}(H)} \cdot \frac{\operatorname{tri}(H)}{3}$. Thus by Observation 2.8 , we may cover $H_{e}$ by $|V(\widetilde{G})|$ balls of radius $r / 2$. This gives a covering of all of $\widetilde{G}^{\ominus k}$ by at most $|E(\widetilde{G})||V(\widetilde{G})|$ balls of radius $r / 2$.

Otherwise, assume $\frac{\operatorname{len}\left(\widetilde{G}^{\ominus k}\right)}{6} \geq r$. Since $s \in B(x, r)$, but $2 r \leq \frac{\operatorname{len}\left(\widetilde{G}^{\ominus k}\right)}{3}$, the ball must be completely contained inside $H_{\left(s, s^{\prime}\right)}$. By induction, we can find a sufficient cover of this smaller graph.

Lemma 2.10. We can cover any ball $B(x, r)$ in $\widetilde{G}^{\ominus k}$ with at most $2|V(\widetilde{G})||E(\widetilde{G})|^{2}$ balls of radius $r / 2$.

Proof. We prove this lemma using induction. For $\widetilde{G}^{\ominus 0}$, the claim holds trivially. Next, if any $H_{e}$ contains all of $B(x, r)$, then by induction we are done. Otherwise, for each $H_{e}$ containing $x, B(x, r)$ contains an endpoint of $e$. Then by Lemma 2.9 , we may cover $H_{e}$ by at most $|E(\widetilde{G})||V(\widetilde{G})|$ balls of radius $r / 2$. For all other edges $e^{\prime}=(u, v), x \notin H_{e^{\prime}}$, so we have:

$$
V\left(H_{e^{\prime}}\right) \cap B(x, r) \subseteq B(v, \max (0, r-d(x, v))) \cup B(u, \max (0, r-d(x, u))) .
$$

Thus, using Lemma 2.9 on both of the above balls, we may cover $V\left(H_{e^{\prime}}\right) \cap B(x, r)$ by at most $2|E(\widetilde{G})||V(\widetilde{G})|$ balls of radius $r / 2$. Hence, in total, we need at most $2|V(\widetilde{G})||E(\widetilde{G})|^{2}$ balls of radius $r / 2$ to cover all of $B(x, r)$. 
Proof of Claim 2.7. First note that $|V(\widetilde{G})|=m(D+1)+2 \lesssim m^{2}$. By Lemma 2.10, we have

$$
\lambda\left(\widetilde{G}^{\ominus k}\right) \leq 2|V(\widetilde{G})||E(\widetilde{G})|^{2} \leq 2|V(\widetilde{G})|^{5} \lesssim m^{10} .
$$

Hence $\log \lambda\left(\widetilde{G}^{\ominus k}\right) \lesssim \log m$.

\section{Lower bound}

For any $\pi \in \operatorname{Aut}(G)$, we define a corresponding automorphism $\tilde{\pi}$ of $\tilde{G}$ by $\tilde{\pi}(s)=s, \tilde{\pi}(t)=t$, $\tilde{\pi}\left(s^{\prime}\right)=s^{\prime}, \tilde{\pi}\left(t^{\prime}\right)=t^{\prime}$, and $\tilde{\pi}\left(v^{(i)}\right)=\pi(v)^{(i)}$ for $v \in V, i \in[D+1]$.

Lemma 3.1. Let $G$ be a vertex transitive graph. Let $f: V(\widetilde{G}) \rightarrow L_{2}$ be an injective mapping and define $\bar{f}: V(\widetilde{G}) \rightarrow L_{2}$ by

$$
\bar{f}(x)=\frac{1}{\sqrt{|\operatorname{Aut}(G)|}}(f(\widetilde{\pi} x))_{\pi \in \operatorname{Aut}(G)} .
$$

Let $\beta$ be such that for every $i \in[D+1]$ there exists a vertical edge $\left(u^{(i)}, v^{(i)}\right)$ with $\left\|\bar{f}\left(u^{(i)}\right)-\bar{f}\left(v^{(i)}\right)\right\| \geq$ $\beta$. Then there exists a horizontal edge $(x, y) \in E(\widetilde{G})$ such that

$$
\frac{\|\bar{f}(x)-\bar{f}(y)\|^{2}}{d_{\widetilde{G}}(x, y)^{2}} \geq \frac{\overline{\| f}(s)-\bar{f}(t) \|^{2}}{d_{\widetilde{G}}(s, t)^{2}}+\frac{\beta^{2}}{36}
$$

Proof. Let $D=\operatorname{diam}(G)$. We first observe three facts about $\bar{f}$, which rely on the fact that when Aut $(G)$ is transitive, for every $x \in V$, the orbits $\{\pi(x)\}_{\pi \in \operatorname{Aut}(G)}$ all have the same cardinality.

(F1) $\|\bar{f}(s)-\bar{f}(t)\|=\|f(s)-f(t)\|$

(F2) For all $u, v \in V$,

$$
\begin{aligned}
\left\|\bar{f}(s)-\bar{f}\left(v^{(1)}\right)\right\| & =\left\|\bar{f}(s)-\bar{f}\left(u^{(1)}\right)\right\|, \\
\left\|\bar{f}(t)-\bar{f}\left(v^{(D+1)}\right)\right\| & =\left\|\bar{f}(t)-\bar{f}\left(u^{(D+1)}\right)\right\| .
\end{aligned}
$$

(F3) For every $u, v \in V, i \in[D]$,

$$
\left\|\bar{f}\left(v^{(i)}\right)-\bar{f}\left(v^{(i+1)}\right)\right\|=\left\|\bar{f}\left(u^{(i)}\right)-\bar{f}\left(u^{(i+1)}\right)\right\| .
$$

(F4) For every pair of vertices $u, v \in V$ and $i \in[D+1]$,

$$
\left\langle\bar{f}(s)-\bar{f}(t), \bar{f}\left(u^{(i)}\right)-\bar{f}\left(v^{(i)}\right)\right\rangle=0 .
$$

Let $z=\frac{\bar{f}(s)-\bar{f}(t)}{\|f(s)-f(t)\|}$. Fix some $r \in V$ and let $\rho_{0}=\left|\left\langle z, \bar{f}(s)-\bar{f}\left(r^{(1)}\right)\right\rangle\right|, \rho_{i}=\left|\left\langle z, \bar{f}\left(r^{(i)}\right)-\bar{f}\left(r^{(i+1)}\right)\right\rangle\right|$ for $i=1,2, \ldots, D$ and $\rho_{D+1}=\left|\left\langle z, \bar{f}(t)-\bar{f}\left(r^{(D+1)}\right)\right\rangle\right|$. Note that, by (F2) and (F3) above, the values $\left\{\rho_{i}\right\}$ do not depend on the representative $r \in V$. In this case, we have

$$
\sum_{i=0}^{D+1} \rho_{i} \geq\|\bar{f}(s)-\bar{f}(t)\|=9 \gamma D,
$$


where we put $\gamma=\frac{\|\bar{f}(s)-\bar{f}(t)\|}{d_{\widetilde{G}}(s, t)}$. Note that $\gamma>0$ since $f$ is injective. then

Recalling that $d_{\widetilde{G}}(s, t)=9 D$ and $d_{\widetilde{G}}\left(s, r^{(1)}\right)=4 D$, observe that if $\rho_{0}^{2} \geq\left(1+\frac{\beta^{2}}{36 \gamma^{2}}\right)(4 \gamma D)^{2}$,

$$
\max \left(\frac{\left.\| \bar{f}(s)-\bar{f}\left(s^{\prime}\right)\right) \|^{2}}{d_{\widetilde{G}}\left(s, s^{\prime}\right)^{2}}, \frac{\left\|\bar{f}\left(s^{\prime}\right)-\bar{f}\left(r^{(1)}\right)\right\|^{2}}{d_{\widetilde{G}}\left(s^{\prime}, r^{(1)}\right)^{2}}\right) \geq \gamma^{2}+\frac{\beta^{2}}{36},
$$

verifying (11). The symmetric argument holds for $\rho_{D+1}$, thus we may assume that

$$
\rho_{0}, \rho_{D+1} \leq 4 \gamma D \sqrt{1+\frac{\beta^{2}}{36 \gamma^{2}}} \leq 4 \gamma D\left(1+\frac{\beta^{2}}{72 \gamma^{2}}\right) .
$$

In this case, by (2), there must exist an index $j \in[D]$ such that

$$
\rho_{j} \geq\left(1-\frac{8 \beta^{2}}{72 \gamma^{2}}\right) \gamma=\left(1-\frac{\beta^{2}}{9 \gamma^{2}}\right) \gamma
$$

Now, consider a vertical edge $\left(u^{(j+1)}, v^{(j+1)}\right)$ with $\left\|\bar{f}\left(u^{(j)}\right)-\bar{f}\left(v^{(j)}\right)\right\| \geq \beta$, and let

$$
u^{\prime}=\bar{f}\left(u^{(j)}\right)+\left\langle z, \bar{f}\left(u^{(j)}\right)-\bar{f}\left(u^{(j+1)}\right)\right\rangle z .
$$

From (F4), and the Pythagorean inequality we have,

$$
\begin{aligned}
& \max \left(\left\|\bar{f}\left(u^{(j)}\right)-\bar{f}\left(u^{(j+1)}\right)\right\|^{2},\left\|\bar{f}\left(u^{(j)}\right)-\bar{f}\left(v^{(j+1)}\right)\right\|^{2}\right)= \\
&\left\|\bar{f}\left(u^{(j)}\right)-u^{\prime}\right\|^{2}\left.+\max \left(\left\|u^{\prime}-\bar{f}\left(u^{(j+1)}\right)\right\|^{2},\left\|u^{\prime}-\bar{f}\left(v^{(j+1)}\right)\right\|^{2}\right)\right) \\
& \geq \rho_{j}^{2}+\frac{\beta^{2}}{4} \\
& \geq\left(1-\frac{2 \beta^{2}}{9 \gamma^{2}}\right) \gamma^{2}+\frac{\beta^{2}}{4} \\
& \geq \gamma^{2}+\frac{\beta^{2}}{36},
\end{aligned}
$$

again verifying (1) for one of the two edges $\left(u^{(j)}, v^{(j+1)}\right)$ or $\left(u^{(j)}, u^{(j+1)}\right)$.

The following lemma is well-known, and follows from the variational characterization of eigenvalues (see, e.g. [15, Ch. 15]).

Lemma 3.2. If $G=(V, E)$ is a d-regular graph with second Laplacian eigenvalue $\mu_{2}(G)$, then for any mapping $f: V \rightarrow L_{2}$, we have

$$
\mathbb{E}_{x, y \in V}\|f(x)-f(y)\|^{2} \lesssim \frac{d}{\mu_{2}(G)} \mathbb{E}_{(x, y) \in E}\|f(x)-f(y)\|^{2}
$$

The next lemma shows that when we use an expander graph, we get a significant increase in stretch for edges of $\widetilde{G}$.

Lemma 3.3. Let $G=(V, E)$ be a d-regular vertex-transitive graph with $m=|V|$ and $\mu_{2}=\mu_{2}(G)$. If $f: V(\widetilde{G}) \rightarrow L_{2}$ is any non-contractive mapping, then there exists a horizontal edge $(x, y) \in E(\widetilde{G})$ with

$$
\frac{\|f(x)-f(y)\|^{2}}{d_{\widetilde{G}}(x, y)^{2}} \geq \frac{\|f(s)-f(t)\|^{2}}{d_{\widetilde{G}}(s, t)^{2}}+\Omega\left(\frac{\mu_{2}}{d}\left(\log _{d} m\right)^{2}\right) .
$$


Proof. We need only prove the existence of an $(x, y) \in E(\widetilde{G})$ such that (4) is satisfied for $\bar{f}$ (as defined in Lemma 3.1), as this implies it is also satisfied for $f$ (possibly for some other edge $(x, y)$ ).

Consider any layer $G^{(i)}$ in $\widetilde{G}$, for $i \in[D+1]$. Applying (3) and using the fact that $f$ is non-contracting, we have

$$
\begin{aligned}
\mathbb{E}_{(u, v) \in E}\left\|\bar{f}\left(u^{(i)}\right)-\bar{f}\left(v^{(i)}\right)\right\|^{2} & =\mathbb{E}_{(u, v) \in E}\left\|f\left(u^{(i)}\right)-f\left(v^{(i)}\right)\right\|^{2} \\
& \gtrsim \frac{\mu_{2}}{d} \mathbb{E}_{u, v \in V}\left\|f\left(u^{(i)}\right)-f\left(v^{(i)}\right)\right\|^{2} \\
& \geq \frac{\mu_{2}}{d} \mathbb{E}_{u, v \in V} d_{G}(u, v)^{2} \\
& \gtrsim \frac{\mu_{2}}{d}\left(\log _{d} m\right)^{2} .
\end{aligned}
$$

In particular, in every layer $i \in[D+1]$, at least one vertical edge $\left(u^{(i)}, v^{(i)}\right)$ has $\left\|\bar{f}\left(u^{(i)}\right)-\bar{f}\left(v^{(i)}\right)\right\| \gtrsim$ $\sqrt{\frac{\mu_{2}}{d}} \log _{d} m$. Therefore the desired result follows from Lemma 3.1 ,

We now to come our main theorem.

Theorem 3.4. If $G=(V, E)$ is a d-regular, m-vertex, vertex-transitive graph with $\mu_{2}=\mu_{2}(G)$, then

$$
c_{2}\left(\widetilde{G}^{\ominus k}\right) \gtrsim \sqrt{\frac{\mu_{2} k}{d}} \log _{d} m
$$

Proof. Let $f: V\left(\widetilde{G}^{\oslash k}\right) \rightarrow L_{2}$ be any non-contracting embedding. The theorem follows almost immediately by induction: Consider the top level copy of $\widetilde{G}$ in $\widetilde{G}^{\ominus k}$, and call it $G_{0}$. Let $(x, y) \in$ $E\left(G_{0}\right)$ be the horizontal edge for which $\|f(x)-f(y)\|$ is longest. Clearly this edge spans a copy of $\widetilde{G}^{\ominus k-1}$, which we call $G_{1}$. By induction and an application of Lemma 3.3, there exists a (universal) constant $c>0$ and an edge $(u, v) \in E\left(G_{1}\right)$ such that

$$
\begin{aligned}
\frac{\|f(u)-f(v)\|^{2}}{d_{\widetilde{G}^{\ominus k}}(u, v)^{2}} & \geq \frac{c \mu_{2}(k-1)}{d}\left(\log _{d} m\right)^{2}+\frac{\|f(x)-f(y)\|^{2}}{d_{\widetilde{G}^{\ominus k}}(x, y)^{2}} \\
& \geq \frac{c \mu_{2}(k-1)}{d}\left(\log _{d} m\right)^{2}+\frac{c \mu_{2}}{d}\left(\log _{d} m\right)^{2}+\frac{\|f(s)-f(t)\|^{2}}{d_{\tilde{G}^{\ominus k}}(s, t)}
\end{aligned}
$$

completing the proof.

Corollary 3.5. If $G=(V, E)$ is an $O(1)$-regular $m$-vertex, vertex-transitive graph with $\mu_{2}=\Omega(1)$, then

$$
c_{2}\left(\widetilde{G}^{\ominus k}\right) \gtrsim \sqrt{k} \log m \approx \sqrt{\log m \log N},
$$

where $N=\left|V\left(\widetilde{G}^{\ominus k}\right)\right|=2^{\Theta(k \log m)}$.

We remark that infinite families of $O(1)$-regular vertex-transitive graphs with $\mu_{2} \geq \Omega(1)$ are well-known. In particular, one can take any construction coming from the Cayley graphs of finitely generated groups. We refer to the survey [6]; see, in particular, Margulis' construction in Section 8. 


\subsection{Extension to other $L_{p}$ spaces}

Our previous lower bound dealt only with $L_{2}$. We now prove the following.

Theorem 3.6. If $G=(V, E)$ is an $O(1)$-regular $m$-vertex, vertex-transitive graph with $\mu_{2}=\Omega(1)$, for any $p>1$, there exists a constant $C(p)$ such that

$$
c_{p}\left(\widetilde{G}^{\ominus k}\right) \gtrsim C(p) k^{1 / q} \log m \approx C(p)(\log m)^{1-1 / q}(\log N)^{1 / q}
$$

were $N=\left|V\left(\widetilde{G}^{\ominus k}\right)\right|$ and $q=\max \{p, 2\}$.

The only changes required are to Lemma 3.2 and Lemma 3.1 (which uses orthogonality). The first can be replaced by Matoušek's [14] Poincaré inequality: If $G=(V, E)$ is an $O(1)$-regular expander graph with $\mu_{2}=\Omega(1)$, then for any $p \in[1, \infty)$ and $f: V \rightarrow L_{p}$,

$$
\mathbb{E}_{x, y \in V}\|f(x)-f(y)\|_{p}^{p} \leq O(2 p)^{p} \mathbb{E}_{(x, y) \in E}\|f(x)-f(y)\|_{p}^{p}
$$

Generalizing Lemma 3.1 is more involved. We need the following well-known 4-point inequalities for $L_{p}$ spaces.

Lemma 3.7. Consider any $p \geq 1$ and $u, v, w, x \in L_{p}$. If $1 \leq p \leq 2$, then

$$
\|u-w\|_{p}^{2}+(p-1)\|x-v\|_{p}^{2} \leq\|u-v\|_{p}^{2}+\|v-w\|_{p}^{2}+\|x-w\|_{p}^{2}+\|u-x\|_{p}^{2} .
$$

If $p \geq 2$, then

$$
\|u-w\|_{p}^{p}+\|x-v\|_{p}^{p} \leq 2^{p-2}\left(\|u-v\|_{p}^{p}+\|v-w\|_{p}^{p}+\|x-w\|_{p}^{p}+\|u-x\|_{p}^{p}\right) .
$$

Proof. The following inequalities are known for $a, b \in L_{p}$ (see, e.g. [3]). If $1 \leq p \leq 2$, then

$$
\left\|\frac{a+b}{2}\right\|_{p}^{2}+(p-1)\left\|\frac{a-b}{2}\right\|_{p}^{2} \leq \frac{\|a\|_{p}^{2}+\|b\|_{p}^{2}}{2} .
$$

On the other hand, if $p \geq 2$, then

$$
\left\|\frac{a+b}{2}\right\|_{p}^{p}+\left\|\frac{a-b}{2}\right\|_{p}^{p} \leq \frac{\|a\|_{p}^{p}+\|b\|_{p}^{p}}{2} .
$$

In both cases, the desired 4-point inequalities are obtained by averaging two incarnations of one of the above inequalities with $a=u-v, b=v-w$ and then $a=u-x, b=x-w$ and using convexity of the $L_{p}$ norm (see, e.g. [12, Lem. 2.1]).

Lemma 3.8. Let $G$ be a vertex transitive graph, and suppose $p>1$. If $q=\max \{p, 2\}$, then there exists a constant $K(p)>0$ such that the following holds. Let $f: V(\widetilde{G}) \rightarrow L_{p}$ be an injective mapping and define $\bar{f}: V(\widetilde{G}) \rightarrow L_{p}$ by

$$
\bar{f}(x)=\frac{1}{|\operatorname{Aut}(G)|^{1 / p}}(f(\widetilde{\pi} x))_{\pi \in \operatorname{Aut}(G)} .
$$

Suppose that $\beta$ is such that for every $i \in[D+1]$, there exists a vertical edge $\left(u^{(i)}, v^{(i)}\right)$ which satisfies $\left\|\bar{f}\left(u^{(i)}\right)-\bar{f}\left(v^{(i)}\right)\right\|_{p} \geq \beta$. Then there exists a horizontal edge $(x, y) \in E(\widetilde{G})$ such that

$$
\frac{\|\bar{f}(x)-\bar{f}(y)\|_{p}^{q}}{d_{\widetilde{G}}(x, y)^{q}} \geq \frac{\|f(s)-f(t)\|_{p}^{q}}{d_{\widetilde{G}}(s, t)^{q}}+K(p) \beta^{q} .
$$


Proof. Let $D=\operatorname{diam}(G)$. For simplicity, we assume that $D$ is even in what follows.

(F1) $\|\bar{f}(s)-\bar{f}(t)\|_{p}=\|f(s)-f(t)\|_{p}$

(F2) For all $u, v \in V$,

$$
\begin{aligned}
\left\|\bar{f}(s)-\bar{f}\left(v^{(1)}\right)\right\|_{p} & =\left\|\bar{f}(s)-\bar{f}\left(u^{(1)}\right)\right\|_{p}, \\
\left\|\bar{f}(t)-\bar{f}\left(v^{(D+1)}\right)\right\|_{p} & =\left\|\bar{f}(t)-\bar{f}\left(u^{(D+1)}\right)\right\|_{p} .
\end{aligned}
$$

(F3) For every $u, v \in V, i \in[D]$,

$$
\left\|\bar{f}\left(v^{(i)}\right)-\bar{f}\left(v^{(i+1)}\right)\right\|_{p}=\left\|\bar{f}\left(u^{(i)}\right)-\bar{f}\left(u^{(i+1)}\right)\right\|_{p} .
$$

Fix some $r \in V$ and let $\rho_{0}=\left\|\bar{f}(s)-\bar{f}\left(r^{(1)}\right)\right\|_{p}, \rho_{i}=\left\|\bar{f}\left(r^{(2 i-1)}\right)-\bar{f}\left(r^{(2 i+1)}\right)\right\|_{p}$ for $i=1, \ldots, D / 2$, $\rho_{D / 2+1}=\left\|\bar{f}(t)-\bar{f}\left(r^{(D+1)}\right)\right\|_{p}$. Also let $\rho_{i, 1}=\left\|\bar{f}\left(r^{(2 i-1)}\right)-\bar{f}\left(r^{(2 i)}\right)\right\|_{p}$ and $\rho_{i, 2}=\| \bar{f}\left(r^{(2 i)}\right)-$ $\bar{f}\left(r^{(2 i+1)}\right) \|_{p}$ for $i=1, \ldots, D / 2$.

Note that, by (F2) and (F3) above, the values $\left\{\rho_{i}\right\}$ do not depend on the representative $r \in V$. In this case, we have

$$
\sum_{i=0}^{D / 2+1} \rho_{i} \geq\|\bar{f}(s)-\bar{f}(t)\|_{p}=9 \gamma D,
$$

where we put $\gamma=\frac{\|f(s)-f(t)\|_{p}}{d_{\widetilde{G}}(s, t)}$. Note that $\gamma>0$ since $f$ is injective.

Let $\delta=\delta(p)$ be a constant to be chosen shortly. Recalling that $d_{\widetilde{G}}(s, t)=9 D$ and $d_{\widetilde{G}}\left(s, r^{(1)}\right)=$ $4 D$, observe that if $\rho_{0}^{q} \geq\left(1+\delta \frac{\beta^{q}}{\gamma^{q}}\right)(4 \gamma D)^{q}$, then

$$
\max \left(\frac{\left.\| \bar{f}(s)-\bar{f}\left(s^{\prime}\right)\right) \|_{p}^{q}}{d_{\widetilde{G}}\left(s, s^{\prime}\right)^{q}}, \frac{\left\|\bar{f}\left(s^{\prime}\right)-\bar{f}\left(r^{(1)}\right)\right\|_{p}^{q}}{d_{\widetilde{G}}\left(s^{\prime}, r^{(1)}\right)^{q}}\right) \geq \gamma^{q}+\delta \beta^{q},
$$

verifying (7). The symmetric argument holds for $\rho_{D / 2+1}$, thus we may assume that

$$
\rho_{0}, \rho_{D / 2+1} \leq 4 \gamma D\left(1+\delta \frac{\beta^{q}}{\gamma^{q}}\right)^{1 / q} \leq 4 \gamma D\left(1+\delta \frac{\beta^{q}}{\gamma^{q}}\right) .
$$

Similarly, we may assume that $\rho_{i, 1}, \rho_{i, 2} \leq \gamma\left(1+\delta \frac{\beta^{q}}{\gamma^{q}}\right)^{1 / q}$ for every $i \in[D / 2]$.

In this case, by (8), there must exist an index $j \in\{1,2, \ldots, D / 2\}$ such that

$$
\rho_{j} \geq\left(1-8 \delta \frac{\beta^{q}}{\gamma^{q}}\right) 2 \gamma
$$

Now, consider a vertical edge $\left(u^{(2 j)}, v^{(2 j)}\right)$ with $\left\|f\left(u^{(2 j)}\right)-f\left(v^{(2 j)}\right)\right\|_{p} \geq \beta$. Also consider the vertices $v^{(2 j-1)}$ and $v^{(2 j+1)}$. We now replace the use of orthogonality ((F4) in Lemma 3.1) with Lemma 3.7 .

We apply one of (5) or (6) of these two inequalities with $x=f\left(u^{(2 j)}\right), v=f\left(v^{(2 j)}\right), u=$ $f\left(v^{(2 j-1)}\right), w=f\left(v^{(2 j+1)}\right)$. In the case $p \geq 2$, we use (5) to conclude that

$$
\begin{aligned}
\left\|f\left(u^{(2 j)}\right)-f\left(v^{(2 j-1)}\right)\right\|_{p}^{p}+\left\|f\left(u^{(2 j)}\right)-f\left(v^{(2 j+1)}\right)\right\|_{p}^{p} & \geq 2^{-p+2} \rho_{j}^{p}+2^{-q+2} \beta^{p}-\rho_{j, 1}^{p}-\rho_{j, 2}^{p} \\
& \geq 2 \gamma^{p}+2^{-p+2} \beta^{p}-34 \delta p \beta^{p} .
\end{aligned}
$$


Thus choosing $\delta=\frac{2^{1-p}}{34 p}$ yields the desired result for one of $\left(u^{(2 j)}, v^{(2 j-1)}\right)$ or $\left(u^{(2 j)}, v^{(2 j+1)}\right)$.

In the case $1 \leq p \leq 2$, we use (6) to conclude that

$$
\left\|f\left(u^{(2 j)}\right)-f\left(v^{(2 j-1)}\right)\right\|_{p}^{2}+\left\|f\left(u^{(2 j)}\right)-f\left(v^{(2 j+1)}\right)\right\|_{p}^{2} \geq \rho_{j}^{2}+(p-1) \beta^{2}-\rho_{j, 1}^{2}-\rho_{j, 2}^{2} .
$$

A similar choice of $\delta$ again yields the desired result.

\section{References}

[1] S. Arora, J. R. Lee, and A. Naor. Euclidean distortion and the Sparsest Cut. J. Amer. Math. Soc., 21(1):1-21, 2008.

[2] Y. Aumann and Y. Rabani. An $O(\log k)$ approximate min-cut max-flow theorem and approximation algorithm. SIAM J. Comput., 27(1):291-301 (electronic), 1998.

[3] K. Ball, E. A. Carlen, and E. H. Lieb. Sharp uniform convexity and smoothness inequalities for trace norms. Invent. Math., 115(3):463-482, 1994.

[4] S. Chawla, A. Gupta, and H. Räcke. An improved approximation to sparsest cut. In Proceedings of the 16th Annual ACM-SIAM Symposium on Discrete Algorithms, Vancouver, 2005. ACM.

[5] A. Gupta, R. Krauthgamer, and J. R. Lee. Bounded geometries, fractals, and low-distortion embeddings. In 44th Symposium on Foundations of Computer Science, pages 534-543, 2003.

[6] S. Hoory, N. Linial, and A. Wigderson. Expander graphs and their applications. Bull. Amer. Math. Soc. (N.S.), 43(4):439-561 (electronic), 2006.

[7] R. Krauthgamer, J. R. Lee, M. Mendel, and A. Naor. Measured descent: A new embedding method for finite metrics. Geom. Funct. Anal., 15(4):839-858, 2005.

[8] T. J. Laakso. Plane with $A_{\infty}$-weighted metric not bi-Lipschitz embeddable to $\mathbb{R}^{N}$. Bull. London Math. Soc., 34(6):667-676, 2002.

[9] U. Lang and C. Plaut. Bilipschitz embeddings of metric spaces into space forms. Geom. Dedicata, 87(1-3):285-307, 2001.

[10] J. R. Lee. On distance scales, embeddings, and efficient relaxations of the cut cone. In SODA '05: Proceedings of the sixteenth annual ACM-SIAM symposium on Discrete algorithms, pages 92-101, Philadelphia, PA, USA, 2005. Society for Industrial and Applied Mathematics.

[11] J. R. Lee. Volume distortion for subsets of Euclidean spaces. Discrete Comput. Geom., 41(4):590-615, 2009.

[12] J. R. Lee and A. Naor. Embedding the diamond graph in $L_{p}$ and dimension reduction in $L_{1}$. Geom. Funct. Anal., 14(4):745-747, 2004.

[13] N. Linial, E. London, and Y. Rabinovich. The geometry of graphs and some of its algorithmic applications. Combinatorica, 15(2):215-245, 1995.

[14] J. Matoušek. On embedding expanders into $l_{p}$ spaces. Israel J. Math., 102:189-197, 1997. 
[15] J. Matoušek. Lectures on discrete geometry, volume 212 of Graduate Texts in Mathematics. Springer-Verlag, New York, 2002.

[16] I. Newman and Y. Rabinovich. A lower bound on the distortion of embedding planar metrics into Euclidean space. Discrete Comput. Geom., 29(1):77-81, 2003.

[17] S. Rao. Small distortion and volume preserving embeddings for planar and Euclidean metrics. In Proceedings of the 15th Annual Symposium on Computational Geometry, pages 300-306, New York, 1999. ACM. 